\title{
Spontaneous Cryptococcal Peritonitis Successfully Treated with Fluconazole
}

\author{
Maysaa Abdallah Saeed ${ }^{1}$ and Tarig S.A. Al Khuwaitir ${ }^{2}$ \\ ${ }^{I}$ Tropical Medicine Department, Faculty of Medicine, Zagazig University, Egypt. \\ ${ }^{2}$ Medicine Department, King Saud Medical City, Kingdom of Saudi Arabia
}

\section{Corresponding Author \\ Maysaa Abdallah \\ Saeed}

Mobile: +2012755949

79

\section{E mail:}

dr.maysaaabdallah@w indowslive.com

Key words: cryptococcal peritonitis; Fluconazole

\section{ABSTRACT}

Spontaneous cryptococcal peritonitis is an uncommon infection that predominantly encountered in immunocompromised. Its clinical presentation is indistinguishable from spontaneous bacterial peritonitis and often results in fatal outcome. Liver disease is an under recognized predisposition for cryptococcal disease. We report a case of spontaneous cryptococcal peritonitis in HIV-negative, non-diabetic female patient with chronic hepatitis $\mathrm{C}$ related cirrhosis. Cryptococcus infection was diagnosed by India ink and culture of ascetic fluid. The patient was successfully treated with fluconazole and she was well at the follow up of 9 months. Early clinical suspicion and initiation of antifungal are of paramount to save the patient's life.

\section{INTRODUCTION}

Spontaneous bacterial peritonitis (SBP) is a common complication in cirrhotic patients with ascites. Clinically it manifests, as fever, abdominal pain and abdominal tenderness. The diagnosis is confirmed by the presence of $>250$ neutrophils $/ \mathrm{mm}^{3}$ in the ascetic fluid and by demonstration of bacteria on Gram stained smear or peritoneal fluid culture [1]. Cryptococcal peritonitis is an uncommon infection and it is rarely reported. Delayed diagnosis of cryptococcal peritonitis due to its rarity and the fact that its presentation is indistinguishable from SBP often results in fatal outcome [2]. We present a case of spontaneous cryptococcal peritonitis in a patient with decompensated cirrhosis who successfully treated with fluconazole.

\section{Case Report}

A 55-years old HIV-negative, nondiabetic, Egyptian female was admitted to King Saud Medical City with complaints of abdominal pain and increasing ascites for two weeks. She was diagnosed as chronic hepatitis C- related cirrhosis. On admission she was afebrile, conscious, had jaundice and bilateral lower limbs edema. Tense ascites and generalized abdominal tenderness were detected on abdominal examination. Her laboratory parameters revealed: total leukocytic count: $6.7 \times 10^{3}$ cells $/ \mathrm{mm}^{3}$ with $75 \%$ neutrophils, hemoglobin: $9 \mathrm{~g} / \mathrm{dl}$, platelets: $90000 / \mathrm{mm}^{3}$, INR: 1.3 , creatinine: $1 \mathrm{mg} / \mathrm{dl}$, total bilirubin: 5.9 $\mathrm{mg} / \mathrm{dl}$ (direct $3.6 \mathrm{mg} / \mathrm{dl}$ ), ALT:65 U/L, ALP: $90 \mathrm{U} / \mathrm{L}$ and serum albumin $2 \mathrm{~g} / \mathrm{dl}$. Abdominal ultrasound revealed cirrhotic liver with ascites. Abdominal paracentesis revealed slightly turbid ascetic fluid, cell count of $600 / \mathrm{mm}^{3}$ with predominant neutrophils $(60 \%)$, albumin level of $0.8 \mathrm{~g} / \mathrm{L}$, glucose: $55 \mathrm{mg} / \mathrm{dl}$ and bacterial culture did not show any growth. Her chest X-ray did not show any infiltrate. Patient was treated empirically with cefotaxime 2 grams every 8 hours. On hospital day three she developed fever so the patient was referred to Infectious Diseases Unit where paracentesis was requested in addition to India ink. Repeated paracentesis revealed similar cytological findings without any decrease in ascetic fluid cell count. India ink preparation showed encapsulated budding yeast cells with morphology suggestive of Cryptococcus neoformans (C. neoformans). Intravenous fluconazole started immediately in a dose of $200 \mathrm{mg}$ once daily. Ascetic fluid culture taken from the first day of admission grew C. neoformans after 9 days incubation. Blood and urine cultures did not reveal growth of any organisms. The patient became afebrile 
3 days after starting fluconazole and showed gradual clinical improvement. Ascetic fluid culture was sterile 3 weeks later. The patient was discharged on oral fluconazole $100 \mathrm{mg}$ once daily and it was continued for 6 months, with careful monitoring of liver function tests. The patient was well at the follow up of 9 months.

\section{DISCUSSION}

Cryptococcus neoformans causes significant infection in immunocompromised individuals with HIV infection, organ transplantation, malignancy and prolonged glucocorticoid therapy $[\mathbf{3 , 4 , 5}]$. The respiratory tract is considered to be the usual portal of entry. However, the GIT has been proposed as a potential site either following ingestion or possible direct inoculation of $C$. neoformans into the blood stream following upper GI bleeding or overgrowth of fungus after antibiotic use $[3,6]$. Our patient, had history of previous antibiotic exposure and upper GI bleeding 2 weeks before development of cryptococcal peritonitis. This situation raise the possibility of translocation of the Cryptococci from GI into the blood stream.

Review of previous reports reveals a striking association between chronic liver disease and cryptococcal peritonitis [7]. Patient with cirrhosis are immunocompromised for several reasons. First, there is a relative complement deficiency leading to reduced opsonic recognition of fungi, as well as deterioration of humoral immunity [2]. In addition, the function of polymorphonuclear leukocyt (PMLs) is reduced [8,9]. The clinical presentation of spontaneous fungal peritonitis (SFP) may be similar to that of spontaneous bacterial peritonitis (SBP) but at times it may be occult. The typical peritoneal cell count is polymorphonuclear predominant ( greater than or equal to $250 \mathrm{cell} / \mathrm{mm}^{3}$ ) but can be mononuclear with lymphocytic predominance [10]. Our patient had decompensated cirrhosis, presented with clinical picture similar to that of SBP and her ascetic fluid analysis revealed $360 \mathrm{PMLs} / \mathrm{mm}^{3}$ which did not show any reduction after 2 days of cefotaxime. The development of fever and the persistence of high PMLs in the ascetic fluid after 2 days of starting antibiotic raise the possibility of SFP in our patient so we asked for India ink preparation after which the diagnosis was confirmed.
Clinician should consider a diagnostic work up and empirical treatment for cryptococcal peritonitis if cell counts in ascetic fluid revealed an elevated leukocyte count but bacterial culture remains negative after 48 hours. Two recent reports in the literatures suggest performing cytology and India ink preparation on ascetic fluid and testing cryptococcal antigen, in the serum and ascetic fluid, may assist in the diagnostic evaluation of patients with suspected cryptococcal peritonitis $[11,12,13]$.

There are no studies evaluating treatment of cryptococcal infection involving sites other than lungs and central nervous system (CNS). In general infection at a single site in the absence of CNS disease, fungemia or risk factors for immunosuppression may be managed with fluconazole for 6-12 months [14]. Our patient had no fungemia and was successfully treated with fluconazole for 6 months. Her ascetic fluid fungal culture was sterile 3 weeks after the index one.

The mortality rate in cirrhotic patients developing cryptococcal peritonitis is high, about $70 \%$ to $80 \%$ of the patients died $[3,15]$. The very poor outcome of these patients is likely to be associated with the underlying advanced liver dysfunction, systemic dissemination and delay in antifungal treatment [10]. In our patient the diagnosis of cryptococcal peritonitis was suspected early and consequently fluconazole started at appropriate time so she was saved. We concluded that Cryptococcus neoformans should be considered as a causative organism for spontaneous peritonitis in cirrhotic patients. Early clinical suspicion and timely initiation of antifungal are of paramount.

\section{REFERENCES}

1- Such J, Runyon BA. Spontaneous bacterial peritonitis. Clin Infec Dis 1998; 27: 669-674.

2- Saif MW, Raj M. Cryptococcal peritonitis complicating hepatic failure: Case report and review of literature. J of Applied Research 2006; 6 (1): 43-50.

3- Mabee CL, Mabee SW, Kirkpatrick RB, Koletar SL. Cirrhosis: a risk factor for cryptococcal peritonitis. Am J Gastroenterol 1995; 90:2042- 2045.

4- Srikanta D, Santiago-Tirado FH, Doering TL. Cryptococcus neoformans: historical curiosity to modern pathogen. Yeast 2014; 31: 47-60. 
5- Negroni R. Cryptococcosis. Clin Dermatol.2012; 30: 599-609.

6- Poblete RB. Cryptococcal peritonitis. Am J Med 1987; 82: 665- 667.

7- Yinnon AM, Solages A, Treanor JJ. Cryptococcal peritonitis: report of a case developing during continuous ambulatory peritoneal dialysis and review of the literature. Clin Infec Dis 1993; 17: 736- 741.

8- Rimola A, Soto R, Arroyo C, Plera C, Rodes J. Reticuloendothelial system phagocytic activity in cirrhosis and its relation to bacterial infections and prognosis. Hepatology 1984; 4: 53-58.

9- Tritto G, Frances R, Shah N, Stadlbauer V, Davis N, Mookeriee RP. Plasma from stable patient with cirrhosis transmit sever neutrophil phagocytic dysfunction which is independent of the presence of bacterial DNA and associated with increased expression of TLR-4. Hepatology 2009; 50: 311A.

10- Park WB, Choe YJ, Lee KD, Lee SC, Kim HB, Kim NJ, et al. Spontaneous cryptococcal peritonitis in patient with liver cirrhosis. Am J Med 2006; 119: 169-171.
11- Albert-Braun S, Venema F, Bausch J, Hunfeld KP, Schafer V. Cryptococcus neoformans peritonitis in a patient with alcoholic cirrhosis: case report and review of the literature. Infection 2005; 33: 282-288.

12- El-Kersh K, Rawasia WF, Chadda U, Guradiola J. Rarity revisited cryptococcal peritonitis. $B M J$ Case Rep published on line. 2014.

13- Jean SS, Fang CT, Shau WY, Chen YC, Shang SC, Hsueh PR, et al. Cryptococcaemia: clinical features and prognostic factors. QJM 2002; 95: 511-518.

14- Perfect JR, Dismukes WE, Dromer F. Clinical practice guidline for the management of cryptococcal diseases: 2010 updated by the infectious diseases society of America.Clin Infect Dis 2010; 50: 291

15- Singh N, Husain S, De Vera M, Gayowski T, Cacciarelli TV. Cryptococcus neoformans infection in patient with cirrhosis, including liver transplant candidates. Medicine (Baltimore) 2004; 83:188192. 